Department of Population Health Sciences, National Institute of Public Health, Oslo, Norway and Department of Health Services, University of Washington, Seattle, USA

Correspondence to: Dr Branko Kopjar, National Institute of Public Health, Department of Population Health Sciences, POB 4404 Torshov, 0403 Oslo, Norway (email: branko.kopjar@ samfunnsmed.uio.no)

\title{
Population preventable fraction of bicycle related head injuries
}

\author{
Branko Kopjar
}

\begin{abstract}
Objective-This study analyzes the population attributable fraction (PAF) of bicycle head injuries due to non-helmet use. Methods-The concept of the PAF and Levin's formula for its calculation were used to develop mathematical models for estimation of: (i) attributable fraction of bicycle related head injuries in the population due to non-helmet use, (ii) expected proportion of helmeted cases among all head injuries, and (iii) estimate of the helmet use rate in the population based on patient case information. The PAF was calculated for a sample of injuries from Stavanger, Norway.

Results-Levin's formula was used to calculate the PAF. Two additional mathematical models were developed for calculating the expected proportion of helmeted cases and the estimation of the helmet use rate in the population. The calculation examples for all models were shown. It was estimated that 133 out of 210 injuries could have been avoided in Stavanger between 1990 and 1996 if all children aged 0-14 had used helmets.

Conclusions-If applied correctly, the PAF is a valid and useful indicator for the population effects of bicycle helmets. The models developed in this study may help to better interpret and predict the population effects of helmet promotion interventions.
\end{abstract}

(Injury Prevention 2000;6:235-238)

Keywords: population attributable risk; head injury; bicycle injury; bicycle helmet

Effectiveness of bicycle safety helmets to prevent head injury has been documented in several case-control studies. ${ }^{1-4}$ Helmets reduce the risk of bicycle related head injury by $60 \%-90 \% .^{12}$ Still, many bicycle riders do not wear helmets. Up to $75 \%$ of the bicycle related head fatalities and approximately $80 \%$ of the bicycle related head injuries in the US that occurred from 1989 to 1992 might have been prevented if the riders had worn helmets. ${ }^{5}$

However not all bicycle related head injuries are preventable by bicycle helmets. What portion of bicycle related head injuries in the population is preventable is influenced by hel- met effectiveness in preventing head injury, and by helmet utilization rate among riders.

This study analyzes the attributable fraction of bicycle injuries in the population due to non-helmet use. The fraction is analyzed as a function of helmet effectiveness and use rate. More specifically, the study provides an estimate of (i) attributable fraction of bicycle related head injuries in the population due to non-helmet use, (ii) expected proportion of helmeted cases among all head injuries, and (iii) estimate of the helmet use rate in the population based on patient case information. The application of the estimation model is shown using empirical data from Stavanger, Norway.

Preventable portion of head injuries

The preventable portion of bicycle related head injuries in the population can be estimated as a population attributable fraction (PAF). ${ }^{6}$ The PAF is the size of the fraction of the incident cases in the population attributable to the exposure. Often, it is used as the estimate of the proportion by which the incidence rate in the population would be reduced if exposure was completely eliminated. According to the PAF, the preventable proportion of bicycle head injuries in the population is equal to:

(Incidence of head injury in all riders)(Incidence of head injury in helmeted riders)

(Incidence of head injury in all riders)

This formula is often not applicable because the required incidences are not known. It would require a major data collection to calculate them. Another way to calculate this proportion is to use Levin's formula. ${ }^{6}$ According to this formula, the attributable fraction of bicycle head injuries in the population is equal to:

$$
\frac{P_{\text {nh }}(R R-1)}{P_{n h}(R R-1)+1}
$$

where, $p_{n h}$ is proportion of riders who do not wear a helmet and, $R R$ is the relative risk of head injury among the non-helmeted riders compared with helmeted riders.

An example of Levin's formula: 


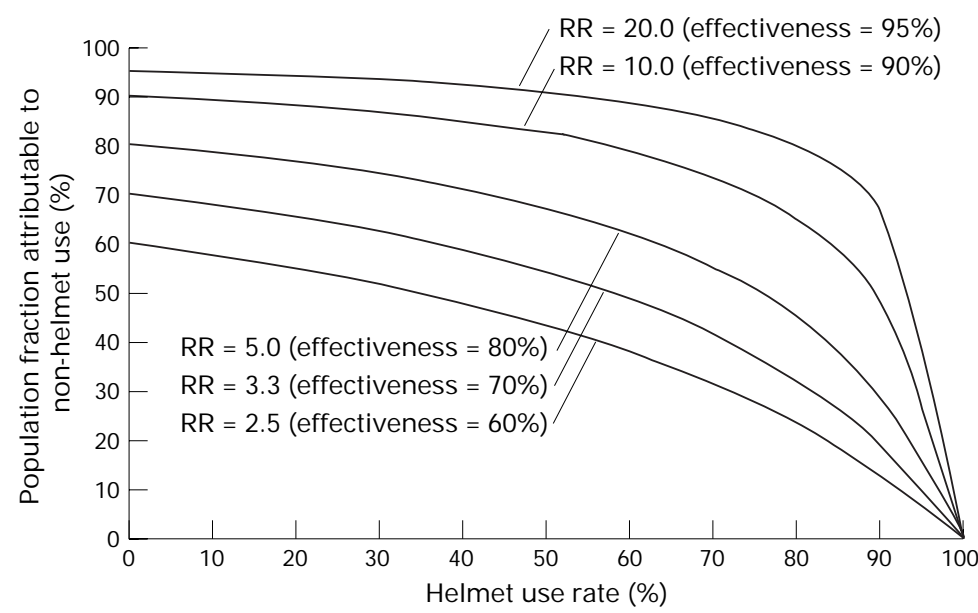

Figure 1 Fraction of bicycle related head injuries attributable to helmet non-use by helmet use rate and helmet effectiveness.

Let's assume that helmets prevent $70 \%$ of all head injuries and that $40 \%$ of the bicycle riders in a hypothetical population use helmets. If helmet effectiveness is $70 \%$, the relative risk of injury among helmeted riders compared with non-helmeted riders is 0.30 . The relative risk among the non-helmeted riders compared with helmeted riders is equal to $0.30^{-1}=3.33$. If $40 \%$ of riders use helmets, the proportion of riders who do not wear helmet is $0.6(60 \%)$. Thus, the fraction of head injuries attributable to non-helmet use in the population is equal to:

$$
\frac{\mathrm{P}_{\mathrm{nh}}(\mathrm{RR}-1)}{\mathrm{P}_{\mathrm{nh}}(\mathrm{RR}-1)+1}=\frac{0.6^{*}(3.33-1)}{0.6(3.33-1)+1}=58 \%
$$

In other words, of 100 bicycle related head injuries occurring in the target population, an estimated 58 would be avoided if every cyclist in the population (and not only $40 \%$ ) had worn a helmet.

Only two pieces of information are required for Levin's formula: the proportion of riders who do not wear a helmet $\left(\mathrm{P}_{\mathrm{nh}}\right)$ and, the relative risk for a non-helmeted rider (RR). Incidence rates of bicycle injuries in the population are not required. Estimation of RR requires cohort or experimental studies of helmet effectiveness that have not yet been performed. However, several case-control studies ${ }^{1-4}$ have reported

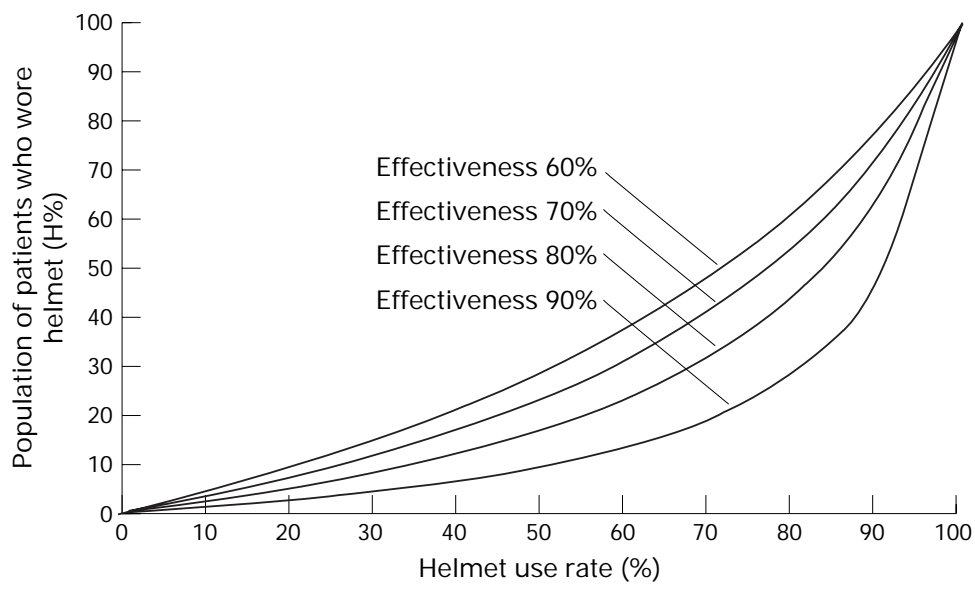

Figure 2 Expected proportion of helmet users among the patients with head injury by helmet use rate and helmet effectiveness. odds ratios (OR) for bicycle helmet effectiveness. A systematic overview of these primary studies investigating bicycle helmet effectiveness is available on the public web site of the Harborview Injury Prevention and Research Center. ${ }^{7}$ Incidence of head injuries is low, suggesting that these ORs can be used as a valid proxy for the RR.

The fraction of bicycle related head injuries attributable to non-helmet use in the population by helmet utilization rate and for various levels of helmet effectiveness is shown in fig 1 . For example, if $50 \%$ of bicycle riders wear helmets, and if helmet reduces the risk of head injury by $60 \%, 47 \%$ of bicycle related head injuries occurring in the population would be prevented had all bicycle riders worn helmets. The slope and the shape of the curve depend on helmet effectiveness. For high RR values, the curve is almost linear and horizontal, except when the use rate reaches high values. For low values of $\mathrm{RR}$, the attributable fraction of the cases decreases relatively rapidly with an increasing helmet use rate. Once the helmet use reaches $100 \%$, all cases of injuries occur to helmeted riders. Attributable fraction is $0 \%$, indicating that no further cases can be prevented.

\section{Probability that a case is helmeted}

Head injuries among bicycle riders occur to helmeted and to non-helmeted riders. The proportion of cases who wore helmets can be calculated by the following formula:

$$
H \%=\frac{P_{h}}{P_{h}+\left(1-P_{h}\right) \times R R} \times 100
$$

where, $H \%$ is a proportion (\%) of helmeted cases, $P_{h}$ is the proportion of riders wearing helmets, and $R R$ is the relative risk of injury among non-helmeted compared with helmeted riders.

This formula has been derived as follows:

$$
\begin{aligned}
H \% & =\frac{\text { No. of helmeted cases }}{\text { No. of helmeted cases }+ \text { No. of non-helmeted cases }} \times 100 \\
& =\frac{P_{h} \times I_{h} \times N}{P_{h} \times I_{h} \times N+P_{n h} \times I_{n h} \times N}=\frac{P_{h} \times I_{h}}{P_{h} \times I_{h}+P_{n h} \times I_{n h}} \\
& =\frac{P_{h} \times I_{h}}{P_{h} \times I_{h}+\left(1-P_{h}\right) \times R R \times I_{h}}=\frac{P_{h}}{P_{h}+\left(1-P_{h}\right) \times R R}
\end{aligned}
$$

where, $I_{h}$ is the incidence among the helmeted riders, $I_{n h}$ is the incidence among the nonhelmeted riders, $P_{n h}$ is the proportion of the riders who do not wear helmet, and $N$ is the size of the population.

Figure 2 shows changes in the percentage of helmeted riders with head injuries $(\mathrm{H} \%)$ by changes in helmet use rate. For example, if $60 \%$ of bicycle riders use a helmet $\left(\mathrm{p}_{\mathrm{h}}=0.6\right)$, and effectiveness is $70 \%(\mathrm{RR}=3.33)$, then $31 \%$ of bicycle related head injuries occurring in the population represents helmet users and $69 \%$ represents non-users. When helmet use rate is high, a significant fraction of the cases represents helmeted riders. These cases are not preventable. 


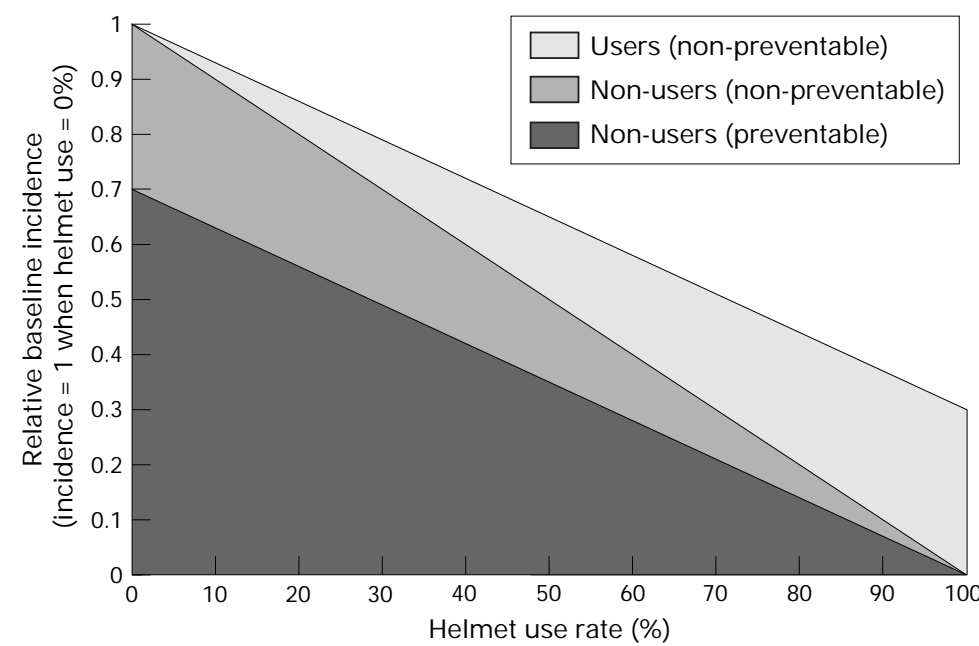

Figure 3 Expected distribution of head injuries by helmet use rate (helmet effectiveness = $70 \%)$.

Table 1 Estimated number of preventable cases of bicycle related bicycle injuries in Stavanger, Norway, 1990-96

\begin{tabular}{lllll}
\hline Age (years) & $\begin{array}{l}\text { Bicycle related head } \\
\text { injuries 1990-96 }\end{array}$ & $\begin{array}{l}\text { Bicycle helmet } \\
\text { utilization rate }\end{array}$ & $P A F^{\star}$ & $\begin{array}{l}\text { Estimated No of } \\
\text { preventable cases }\end{array}$ \\
\hline $0-2$ & 3 & $0 \%$ & $70 \%$ & 2 \\
$3-11$ & 156 & $30 \%$ & $62 \%$ & 97 \\
$12-14$ & 51 & $15 \%$ & $66 \%$ & 34 \\
All ages & 210 & & & 133
\end{tabular}

${ }^{\star}$ Assumed $\mathrm{RR}=3.3(70 \%$ risk reduction), and helmet utilization rate $=100 \%$.

Figure 2 offers a possibility to estimate a helmet use rate in the population, based on a representative case series of head injuries among cyclists with known helmet use status. For example, if $11 \%$ of the cases wore helmets, and helmet effectiveness is $70 \%$, an estimated $30 \%$ of the riders in the population use helmets.

\section{Distribution of cases in the population}

Cases of head injury occurring in the population fall into three categories: (1) injuries among helmet users (non-preventable), (2) preventable cases among non-users, and (3) nonpreventable cases occurring among non-users.

The incidences of head injuries in the population by these three categories can be estimated as follows:

Category (1): $I_{U N P}=I_{\mathrm{B}}{ }^{\times} P_{U} \times R R^{-1}$

Category (2): $I_{N U P}=I_{B} \times P_{N U} \times\left(1-R R^{-1}\right)$

Category (3): $I_{N U N P}=I_{B} \times P_{N U} \times R R^{-1}$

where, $I_{U N P}$ is the incidence rate among users (non-preventable cases among users), $I_{N U P}$ is the incidence rate of preventable cases among non-users, $I_{N U N P}$ is the incidence rate of non-preventable cases among non-users, $I_{B}$ is the background incidence rate in the population (that is, the incidence rate when no one uses helmet), $P_{U}$ is the proportion of bicycle riders who use helmet, $P_{N U}$ is the proportion of bicycle riders who do not use helmets, and $R R$ is the relative risk of injury among nonhelmeted compared with helmeted riders.

If the incidence rate is not available it can be substituted by 1 or by some other index value. That enables calculation of relative distribution of the cases in the population by these three categories. Figure 3 shows the relative distribution of bicycle related head injuries in the population by these three categories. The background incidence rate on the figure has been substituted by 1 . Changes in the incidence rate are directly proportional to the changes in the proportion of riders who wear helmets, assuming the exposure and helmet effectiveness remains constant.

Bicycle related head injuries in Stavanger The applicability of the model is shown on data from the prospective ongoing injury registration system operated by the Central Hospital and emergency ward in Stavanger, Norway. The registration system is described in detail elsewhere. $^{89}$ We selected all bicycle related upper head injuries (injuries to scalp, forehead, and brain) occurring to children from Stavanger aged 0-14 years from 1990 to 1996 $(n=210)$. Helmet effectiveness in preventing upper head injury was assumed to be $70 \%$ $(\mathrm{RR}=3.33)$. Use rate estimates for these children are based on a national survey. ${ }^{10}$ Table 1 shows that approximately $133(63 \%)$ of these injuries would be avoided if all children wore a helmet every time they rode a bike.

\section{Discussion}

The PAF has been used for many years to help in making health policy decisions. The PAF is a useful indicator but it has significant limitations. Researchers frequently make computational mistakes and misinterpretations when using PAF. ${ }^{11}$ The most common misinterpretation is that the PAF represents the true preventable portion of disease. In most situations, the PAF overestimates the true preventive potential because there are competing risks and confounding factors. Researchers and policy makers should use the PAF with great caution and only as an indication of potential benefits of health interventions. True benefits of interventions can be reliably estimated only from intervention studies. The validity of the PAF estimates is also an issue. The validity of PAF estimates depends on the validity of estimates used in the calculation of PAF. In the case of bicycle helmets these are helmet use rates and helmet effectiveness (RR). Bicycle helmets protect from head injuries, but the exact level of protection is unknown. The available estimates of helmet effectiveness ${ }^{1-4}$ are based on observational studies only, and are open for many possible sources of bias and confounding. PAF estimates can be adjusted for confounding ${ }^{12}$ provided that the information about cofounders is available. This is rarely the case in studies of bicycle helmets. Probably the best approach, given the current state of knowledge, is to apply a range of effectiveness estimates, as shown in the current study.

The validity of estimates of current helmet use affects the validity of PAF estimates. If the use rate is overestimated, the PAF will underestimate the preventive potential and vice versa. Use rates are estimated from samples and can be inaccurate. If PAF estimates are used to make global policy decisions, modest inaccuracies in use rate estimates are unlikely to significantly impact the policy decision. For 
other applications of the PAF, inaccuracies may have a substantial impact. An additional issue is the definition of helmet user. A portion of riders use helmets only sometimes. Depending how these users are classified (into users or non-users) a degree of misclassification bias on exposure will be introduced in the estimates and the PAF will under or over-estimated.

The PAF is usually calculated for a total effect attributable to a specific factor. That is an unrealistic expectation for the interventions to promote bicycle helmets. Such interventions can improve use rates up to $40 \%$ over baseline levels, ${ }^{7}$ and that only among children. Our study offers a simple way of estimating effects associated with different levels of increase in helmet use rates.

In spite of the above limitations, the PAF is an appropriate and useful measure for interventions to promote helmet use. The protective effects of helmets are well established, the effect is not confounded by previous non-use, and there is no evidence of competing risks replacing the ones removed by helmet use. These conditions are the basic epidemiologic criteria for external validity of PAF estimates. ${ }^{11}$

\section{Implications for prevention}

An increasing number of bicycle riders around the world benefit from the protective effect of bicycle safety helmets. The changes in helmet use rates influence the occurrence of bicycle injuries in the population. This study helps researchers and program personnel to better interpret and predict these changes and to improve estimates of the potential effectiveness of helmet promotion interventions.

1 Thompson DC, Rivara FP, Thomspon RS. Effectiveness of bicycle safety helmets in preventing head injuries. $7 A M A$ 1996;276:1968-73.

2 Thomas S, Acton C, Nixon J, et al. Effectiveness of bicycle helmets in preventing head injury: case-control study. BMF 1994;308:173-6.

3 Maimaris C, Summers CL, Browning C, et al. Injury patterns in cyclists attending an accident and emergency department: a comparison of helmet wearers and nonwearers. BMf 1994;308:1537-40.

4 Thompson RS, Rivara FP, Thompson DC. A case-control study on the effectiveness of bicycle safety helmets. $N$ Engl f Med 1989;320:1361-7.

5 Sosin DM, Sacks JJ, Webb KW. Pediatric head injuries and deaths from bicycling in the United States. Pediatrics 1996; 98:868-70.

6 Gordis L. Epidemiology. Philadelphia: WB Saunders, 1996.

$7 \mathrm{http} / / /$ depts.washington.edu/hiprc/childinjury/topic/ bicycles/helmeteffect.html

8 Kopjar B, Wickizer T. Cycling to school-a significant health risk? Inj Prev 1995;1:238-41.

9 Kopjar B, Wickizer T. How safe are day-care centers? Daycare versus home injuries among children in Norway. Pediatrics 1996;97:43-7.

10 Institute of Transport Economics. The bicycle survey 1992. Oslo: Institute of Transport Economics (217/1993).

11 Rockhill B, Newman B, Weinberg C. Use and misuse of population attributable fractions. Am f Public Health 1998; 88:15-19.

12 Coughlin SS, Benichou J, Weed DL. Attributable risk estimation in case-control studies. Epidemiol Rev 1994;16: $51-64$.

\section{Duty of care and sport safety equipment}

A Melbourne school baseball player has lost a damages relating to a teacher's duty of care. An exclusive girl's school was cleared of responsibility after a pupil was hit in the face playing baseball. The 18 year old student sued the school for $\$ 20000$ (Aus) after she was hit in the face during baseball practice causing two front teeth to be loosened and ongoing dental treatment. The magistrate found that a supervisor was not in breach of her duty of care in telling the student not to wear a face mask during a training drill and dismissed the complaint (The Age, 24 December 1999). 